\title{
Using Python for Analysis and Verification of Mixed-mode Signal Chains
}

\author{
Mark Thoren ${ }^{\ddagger}$, Cristina Suteu ${ }^{\ddagger *}$
}

Abstract-Any application involving sensitive measurements of the physical world starts with accurate, precise, and low-noise signal chain. Modern, highly integrated data acquisition devices can often be directly connected to sensor outputs, performing analog signal conditioning, digitization, and digital filtering on a single silicon device, greatly simplifying system electronics. However, a complete understanding of the signal chain's noise sources and noise limiting filters is still required to extract maximum performance from and debug these modern devices.

\section{Introduction}

Mixed-mode signal chains are everywhere. Simply put, any system that transforms a real-world signal to an electrical representation, which is then digitized can be classified as a mixed-mode signal chain. At every point along the chain the signal is degraded in various ways that can usually be characterized either as some form of distortion or additive noise. Once in the digital domain, the processing of the digitized data is not perfect either, but at least it is, for all practical purposes, immune to many of the offenders that affect analog signals - component tolerances, temperature drift, interference from adjacent signals or supply voltage variations.

As the industry continues to push the physical limits, one thing that can be stated with certainty is this: there is always room for improvement in analog and mixed signal components for instrumentation. If an Analog to Digital Converter (ADC) or a Digital to Analog Converter (DAC) appears on the market that advances the state of the art in speed, noise, power, accuracy, or price, industry will happily apply it to existing problems, then ask for more improvement. However, in order to achieve the best acquisition system for your application, it is fundamental to be aware of the components' limitations and choose these accordingly.

This tutorial is in extension of Converter Connectivity Tutorial $^{1}$ and associated code and simulation files ${ }^{2}$. A representative signal chain will be analyzed and tested, focusing on noise. Individual signal chain elements will first be modelled with the help of Python / $\mathrm{SciPy}^{3}$ and LTspice ${ }^{4}$, then verified using Python to drive low-cost instrumentation and evaluation boards via the Linux Industrial Input Output (IIO) framework. While primarily for the education space, these instruments have adequate

+ Analog Devices, Inc.

* Corresponding author: cristina.suteu@analog.com

Copyright $@ 2021$ Mark Thoren et al. This is an open-access article distributed under the terms of the Creative Commons Attribution License, which permits unrestricted use, distribution, and reproduction in any medium, provided the original author and source are credited.

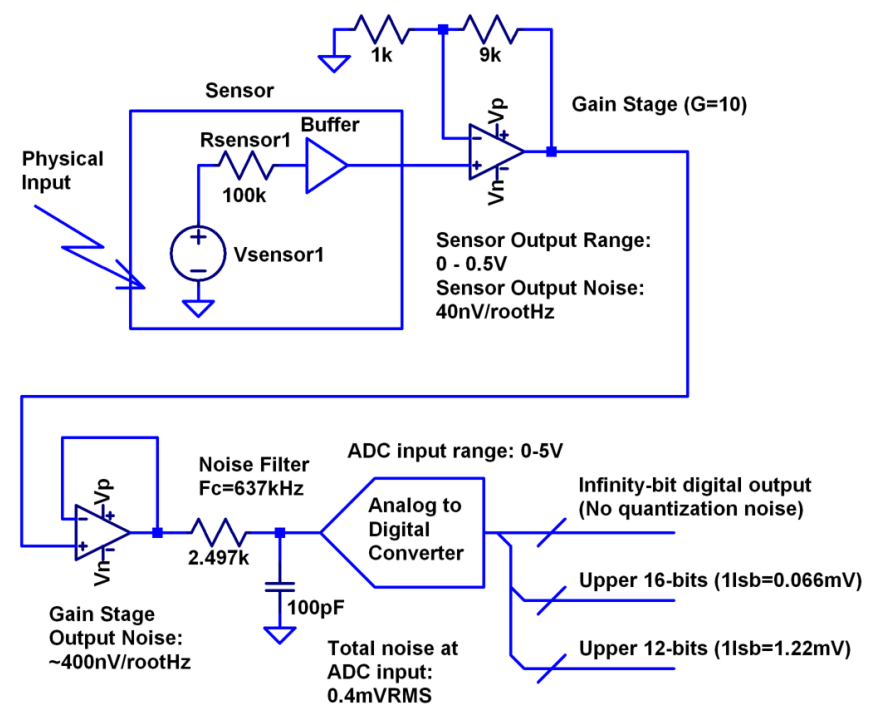

Fig. 1: In a mixed-mode signal chain, some physical phenomenon such as temperature, light intensity, $\mathrm{pH}$, force, or torque is converted to an electrical parameter (resistance, current, or directly to voltage). This signal is then amplified, low-pass filtered, and digitized by an $A D C$, which may include internal digital filtering.

performance for many industrial applications. Furthermore, these techniques can easily be adapted to other bench-top instruments.

\section{A Generic Mixed Signal Chain}

Figure 1 shows a generic signal chain typical of a precision instrumentation application, with a physical input and digital output. There are numerous background references on analog to digital converters available ${ }^{5}$, and most readers will have a sense that an analog to digital converter samples an input signal at some point in time (or measures the average of a signal over some observation time), and produces a numerical representation of that signal - most often as a binary number with some value between zero and $2^{N}-1$ where $N$ is the number of bits in the output word.

\section{ADC Noise Sources}

While there are several noise sources in Figure 1, one that is often either ignored, or over-emphasized, is the number of bits in the ADC's digital output. Historically, an ADC's "number of bits" was considered the ultimate figure of merit, where a 16-bit converter was 4 times better than a 14-bit converter ${ }^{6}$. But in the case of 


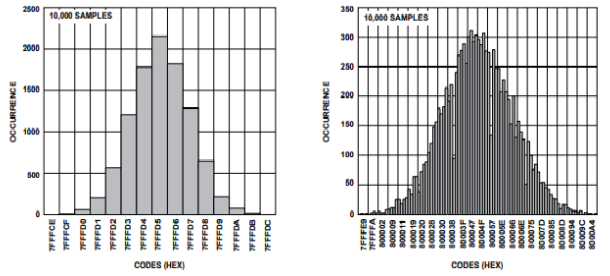

Fig. 2: At a PGA gain of one (left), 13 codes are represented in the AD7124 output noise, and the standard deviation is about 2.5 codes. While quantization is visible, thermal noise is more significant. At a PGA gain of 128 (right), 187 codes are represented, quantization noise is insignificant. Truncating one or two least-significant bits (doubling or quadrupling quantization noise) would not result in a loss of information.

modern, high-resolution converters, the "number of bits" can be safely ignored. Note a general principle of signal chain design:

"The input noise of one stage should be somewhat lower than the output noise of the preceding stage."

As with any signal chain, one noise source within an ADC often dominates. Thus, if a noiseless signal applied to an N-bit ADC:

- results in either a single output code, or two adjacent output codes, then quantization noise dominates. The Signal to Noise Ratio can be no greater than $(6.02 \mathrm{~N}+$ 1.76) $\mathrm{dB}^{7}$.

- results in a gaussian distribution of "many" output codes, then thermal noise source dominates. The Signal to Noise Ratio is no greater than:

$20 \log \left(V_{\text {in }}(p-p) /(\sigma / \sqrt{8})\right)$, where:

$V_{\text {in }}(p-p)$ is the full-scale input signal

$\sigma$ is the standard deviation of the output codes in units of voltage.

Very high resolution converters, such as the AD7124-8 that will be used as an example shortly, are rarely limited by quantization noise; thermal noise dominates in all of the gain / bandwidth settings, and a shorted input will always produce a fairly Gaussian distribution of output codes. Figure 2 , from Ref. ${ }^{8}$ shows the grounded-input histogram of the AD712482, 24-bit sigma-delta ADC, with the internal Programmable Gain Amplifier (PGA) set to 1 and 128 , respectively.

\section{Modeling and Measuring ADC noise}

Modeling the noise of a thermal-noise limited ADC's is straightforward. If the noise is "well behaved" (Gaussian, as it is in Figure 2) and constant across the ADC's input span, the ADC's time-domain noise can be modelled using NumPy's ${ }^{9}$ random. normal function, then verified by taking the standard deviation, as seen in the Model Gaussian Noise code block.

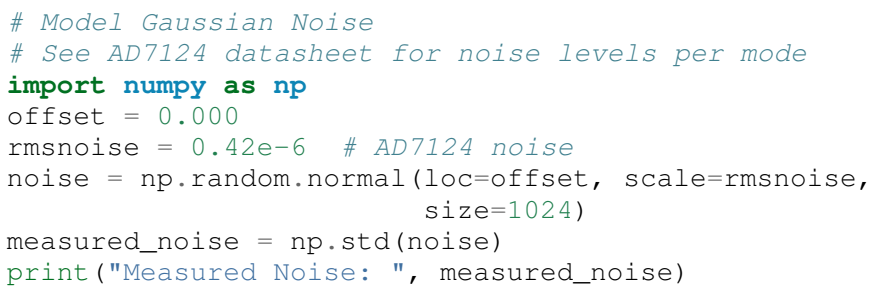

Figure 3 shows the general setup for testing ADC noise and filter response ${ }^{1}$.

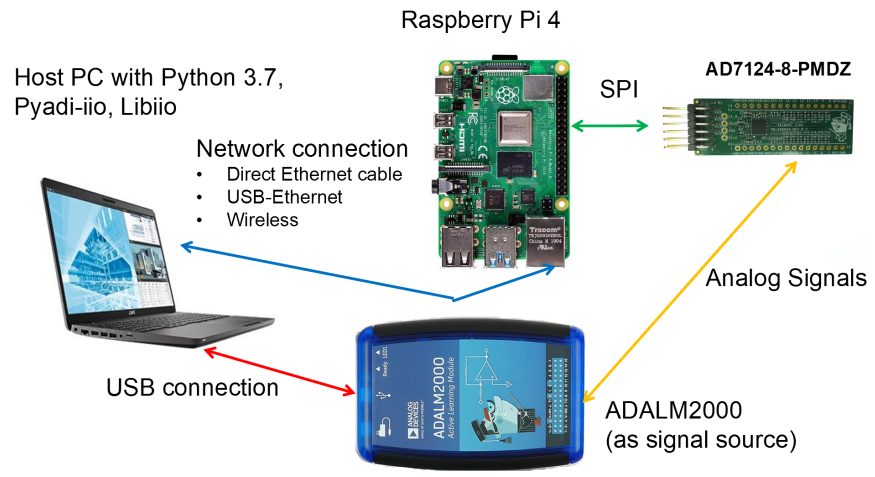

Fig. 3: The ADALM2000 is a multifunction USB test instrument with two general-purpose analog inputs and two outputs, with sample rates of 100Msps and 150Msps, respectively. It can be used as a simple signal source for measuring ADC noise bandwidth and filter response. A Raspberry Pi 4 running a kernel with AD7124 device driver support acts as a simple bridge between the AD7124 and a host computer.

The AD7124 device driver falls under the industry-standard IIO framework, which has a well-established software API (including Python bindings). Application code can run locally (on the Raspberry $\mathrm{Pi}$ ) or on a remote machine via network, serial, or USB connection. Furthermore, the pyadi-iio ${ }^{10}$ abstraction layer takes care of much of the boilerplate setup required for interfacing with IIO devices, greatly simplifying the software interface. The AD7124-8 Basic Data Capture code block illustrates how to open a connection to the AD7124-8, configure it, capture a block of data, then close the connection.

\# AD7124-8 Basic Data Capture

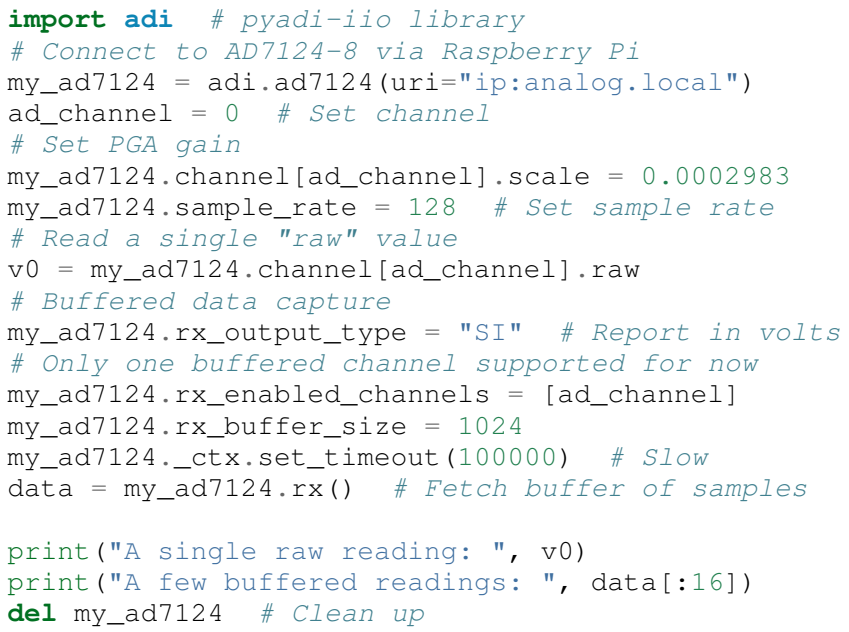

With communication to the AD7124-8 established, an extremely simple, yet extremely useful test can be performed: measuring input noise directly. Simply shorting the input to an ADC and looking at the resulting distribution of ADC codes is a valuable step in characterizing a signal chain design. The AD7124 input mode is set to unipolar, so only positive values are valid; the test circuit shown in Figure 4 ensures that the input is always positive.

Figure 5 shows two, 1024-point measurements. The lower (blue) trace was taken immediately after initially applying power.

The "wandering" can be due to a number of factors - the internal reference warming up, the external resistors warming up (and hence drifting), or parasitic thermocouples, where slightly dissimilar metals will produce a voltage in the presence of 


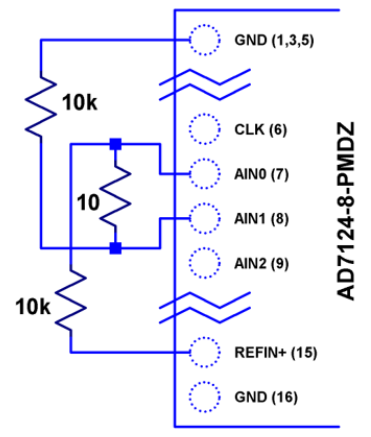

Fig. 4: A resistor divider is used to generate a $1.25 \mathrm{mV}$ bias across the AD7124-8's input, overcoming the $15 \mu \mathrm{V}$ maximum offset voltage and ensuring that $A D C$ readings are always positive.

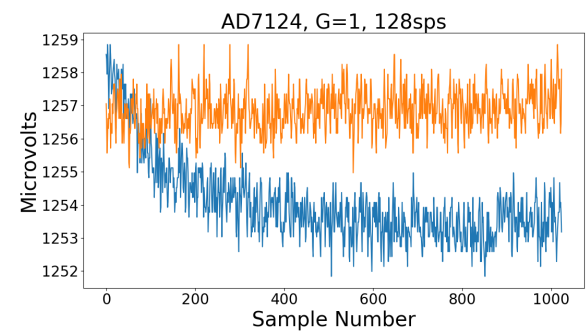

Fig. 5: Two AD7124-8 data captures are taken with a 1.25mV bias applied. The lower trace shows initial drift after power-up as the circuit warms up. The upper trace shows stable readings after a halfhour warmup time.

thermal gradients. Measured noise after warmup is approximately 565nVRMS - on par with the datasheet noise specification.

\section{Expressing ADC Noise as a Density}

The general principle of analog signal chain design (that the input noise of one stage should be somewhat lower than the output noise of the preceding stage) is an easy calculation if all elements include noise density specifications, as most wellspecified sensors, and nearly all amplifiers do.

Unlike amplifiers and sensors, ADC datasheets typically do not include a noise density specification. Expressing the ADC's noise as a density allows it to be directly compared to the noise at the output of the last element in the analog signal chain, which may be an ADC driver stage, a gain stage, or the sensor itself.

An ADC's internal noise will necessarily appear somewhere between DC and half the sample rate. Ideally this noise is flat, or at least predictably shaped. In fact, since the ADC's total noise is spread out across a known bandwidth, it can be converted to a noise density that can be directly compared to other elements in the signal chain. Precision converters typically have total noise given directly, in volts RMS: $e_{R M S}=\sigma$, where $e_{R M S}$ is the total RMS noise, calculated from the standard deviation of a groundedinput histogram of codes.

Higher speed converters that are tested and characterized with sinusoidal signals will typically have a signal to noise (SNR) specification. If provided, the total RMS noise can be calculated as:

$$
e_{R M S}=\frac{A D C p-p}{\sqrt{8} * 10^{\frac{S N R}{20}}}
$$

Where $A D C p-p$ is the peak-to-peak input range of the ADC.

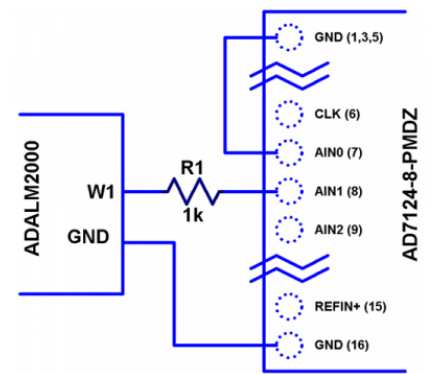

Fig. 6: An ADALM2000 waveform generator is used to generate a range of sinewave frequencies, allowing the AD7124-8's filer response to be measured directly. While the script sets the sinewave amplitude and offset to a safe level, a $1 \mathrm{k}$ resistor protects the AD7124-8 in the event of a malfunction. (The ADALM2000 output voltage range is $-5 \mathrm{~V}$ to $+5 \mathrm{~V}$, while the AD7124-8 absolute maximum limits are $-0.3 \mathrm{~V}$ and $+3.6 \mathrm{~V}$.)

The equivalent noise density can then be calculated:

$$
e_{n}=\frac{e_{R M S}}{\sqrt{\frac{f s}{2}}}
$$

Where $f s$ is the ADC sample rate in samples/second.

The total noise from Figure 5 after warmup was $565 \mathrm{nV}$ at a data rate of 128 sps. The noise density is approximately:

$$
565 \mathrm{nV} / \sqrt{64 \mathrm{~Hz}}=70 \mathrm{nV} / \sqrt{\mathrm{Hz}}
$$

The ADC can now be directly included in the signal chain noise analysis, and leads to a guideline for optimizing the signal chain's gain:

Increase the gain just to the point where the noise density of the last stage before the ADC is a bit higher than that of the ADC, then stop. Don't bother increasing the signal chain gain any more - you're just amplifying noise and decreasing the allowable range of inputs.

This runs counter to the conventional wisdom of "filling" the ADC's input range. There may be benefit to using more of an ADC's input range if there are steps or discontinuities in the ADC's transfer function, but for "well behaved" ADCs (most sigma delta ADCs and modern, high-resolution Successive Approximation Register (SAR) ADCs), optimizing by noise is the preferred approach.

\section{Measuring ADC filter response}

The AD7124-8 is a sigma-delta ADC, in which a modulator produces a high sample rate, but noisy (low resolution), representation of the analog input. This noisy data is then filtered by an internal digital filter, producing a lower rate, lower noise output. The type of filter varies from $\mathrm{ADC}$ to $\mathrm{ADC}$, depending on the intended end application. The AD7124-8 is general-purpose, targeted at precision applications. As such, the digital filter response and output data rate are highly configurable. While the filter response is well-defined in the datasheet, there are occasions when one may want to measure the impact of the filter on a given signal. The AD7124-8 Filter Response code block measures the filter response by applying sinewaves to the ADC input and analyzing the output. This method can be easily adapted to measuring other waveforms - wavelets, simulated physical events. The ADALM2000 is connected to the AD7124-8 circuit as shown in Figure 6. 


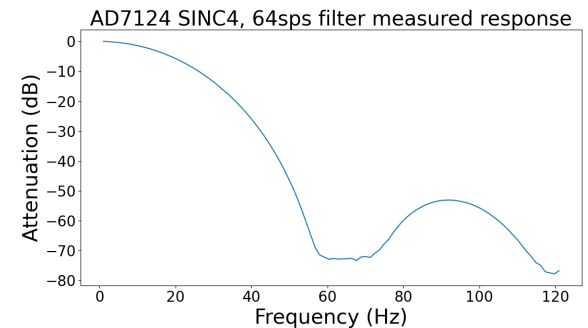

Fig. 7: A measurement of the AD7124 filter response in 64sps, SINC4 mode shows the filter's passband, first lobe, and first two nulls.

The AD7124-8 Filter Response code block will set the ADALM2000's waveform generator to generate a sinewave at $10 \mathrm{~Hz}$, capture 1024 data points, calculate the RMS value, then append the result to a list. (The send_sinewave and capture_data are utility functions that send a sinewave to the ADALM2000 and receive a block of data from the AD7124, respectively ${ }^{2}$.) It will then step through frequencies up to $250 \mathrm{~Hz}$, then plot the result as shown in Figure 7.

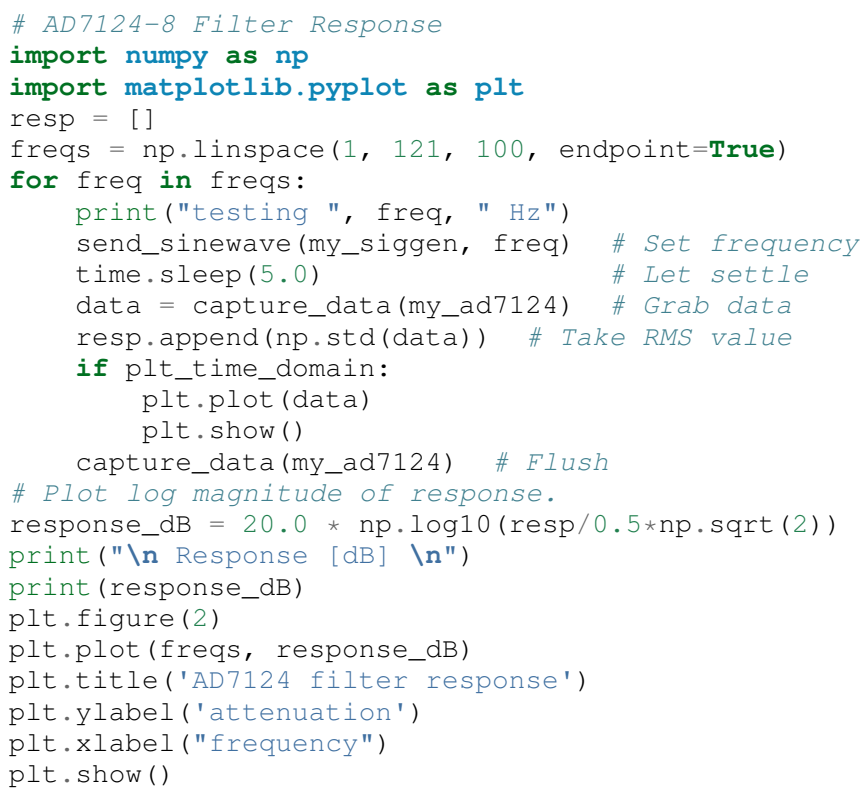

While measuring high attenuation values requires a quieter and lower distortion signal generator, the response of the first few major "lobes" is apparent with this setup.

\section{Modeling ADC filters}

The ability to measure an ADC's filter response is a practical tool for bench verification. However, in order to fully simulate a signal chain, a model of the filter is needed. This isn't explicitly provided for many converters (including the AD7124-8), but a workable model can be reverse engineered from the information provided in the datasheet.

Note that what follows is only a model of the AD7124-8 filters, it is not a bit-accurate representation. Refer to the AD7124-8 datasheet for all guaranteed parameters.

The AD7124's filters all have frequency responses that are combinations of various SINC functions (with a frequency response proportional to $\left.(\sin f / f)^{N}\right)$. These filters are fairly easy to construct, and to reverse-engineer when nulls are known.

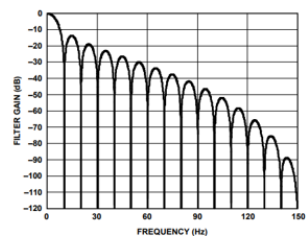

Fig. 8: The AD7124-8 10Hz notch filter has a SINC1 magnitude response; the filter's impulse response is simply an unweighted (rectangular) average of samples over a 100ms time interval.

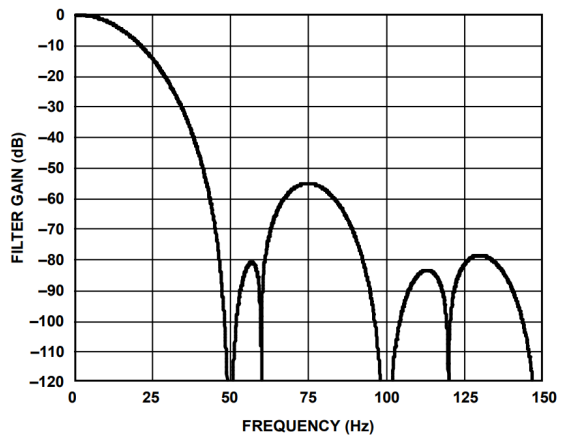

Fig. 9: The AD7124-8 50/60Hz rejection filter response is the combination of a $50 \mathrm{~Hz}$, SINC3 filter and a $60 \mathrm{~Hz}$, SINCl filter.

Figure 8 from Ref. ${ }^{8}$ shows the AD7124-8's $10 \mathrm{~Hz}$ notch filters. Various combinations of higher-order SINC3 and SINC4 filters are also available.

The simultaneous $50 \mathrm{~Hz} / 60 \mathrm{~Hz}$ rejection filter shown in Figure 9, from Ref. ${ }^{8}$ is a nontrivial example. This filter is intended to strongly reject noise from A.C. power lines, which is either $50 \mathrm{~Hz}$ (as in Europe) or $60 \mathrm{~Hz}$ (as in the United States).

Higher order SINC filters can be generated by convolving SINC1 filters. For example, convolving two SINC1 filters (with a rectangular impulse response in time) will result in a triangular impulse response, and a corresponding SINC2 frequency response. The AD7124 Filters code block generates a SINC3 filter with a null at $50 \mathrm{~Hz}$, then adds a fourth filter with a null at $60 \mathrm{~Hz}$.

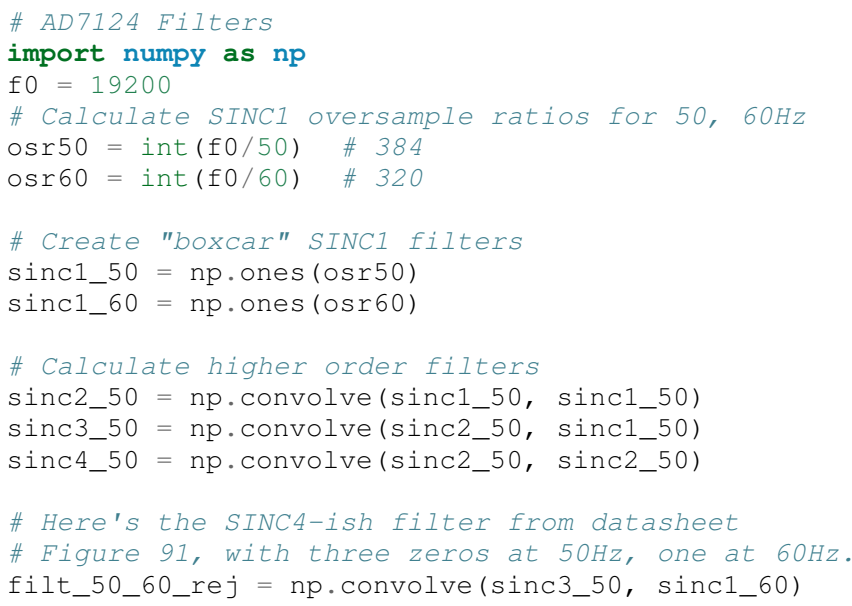

The resulting impulse (time domain) shapes of the filters are shown in Figure 10. Filter coefficient (tap) values are normalized for unity $(0 \mathrm{~dB})$ gain at zero frequency.

And finally, the frequency response can be calculated using NumPy's freqz function, as seen in the AD7124 Frequency Response code block. The response is shown in Figure 11. 


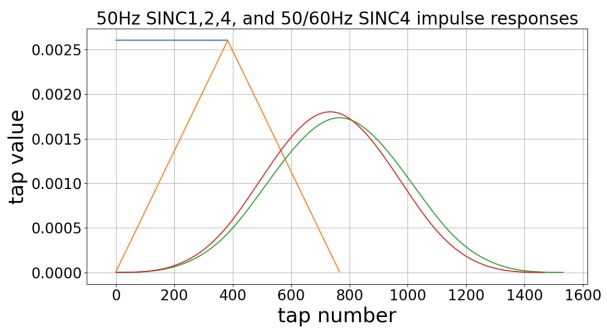

Fig. 10: Repeatedly convolving rectangular impulse responses produces triangular, then "Gaussian-like" impulse responses.

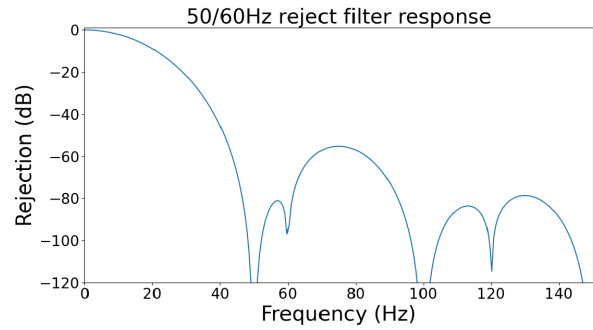

Fig. 11: Convolving a SINC3, $50 \mathrm{~Hz}$ notch filter with a SINC1, $60 \mathrm{~Hz}$ filter produces a composite response that strongly rejects both $50 \mathrm{~Hz}$ and $60 \mathrm{~Hz}$.

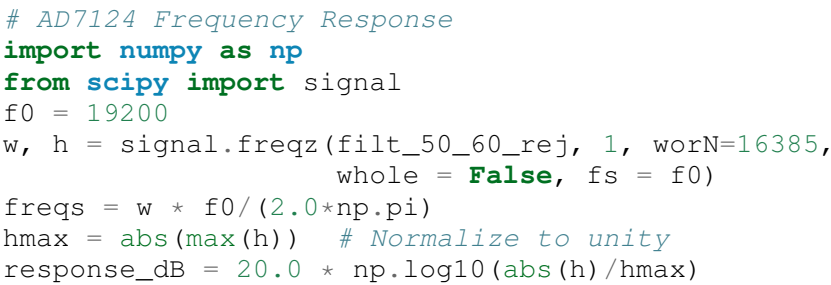

\section{Resistance is Futile: A Fundamental Sensor Limitation}

All sensors, no matter how perfect, have some maximum input value (and a corresponding maximum output - which may be a voltage, current, resistance, or even dial position) and a finite noise floor - "wiggles" at the output that exist even if the input is perfectly still. At some point, a sensor with an electrical output will include an element with a finite resistance (or more generally, impedance) represented by Rsensor in Figure 12. This represents one fundamental noise limit that cannot be improved upon - this resistance will produce, at a minimum:

$e_{n}(R M S)=\sqrt{4 * K * T * \text { Rsensor } *(F 2-F 1)}$ Volts of noise, where:

$e_{n}(R M S)$ is the total noise

$K$ is Boltzmann's constant $(1.38 \mathrm{e}-23 \mathrm{~J} / \mathrm{K})$

$\mathrm{T}$ is the resistor's absolute temperature (Kelvin)

F2 and F1 are the upper and lower limits of the frequency band of interest.

Normalizing the bandwidth to $1 \mathrm{~Hz}$ expresses the noise density, in $\frac{V}{\sqrt{H z}}$.

A sensor's datasheet may specify a low output impedance (often close to zero Ohms), but this is likely a buffer stage - which eases interfacing to downstream circuits, but does not eliminate noise due to impedances earlier in the signal chain.

There are numerous other sensor limitations - mechanical, chemical, optical, each with their own theoretical limits and whose

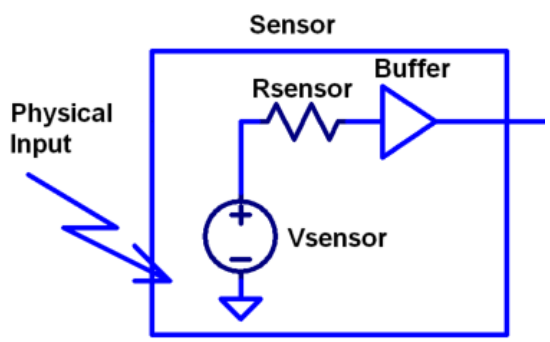

Fig. 12: Sensors often include an internal buffer to simplify connection to downstream circuits. While the output impedance is low (often approaching zero Ohms), noise from high impedance sensing elements is buffered along with the signal.

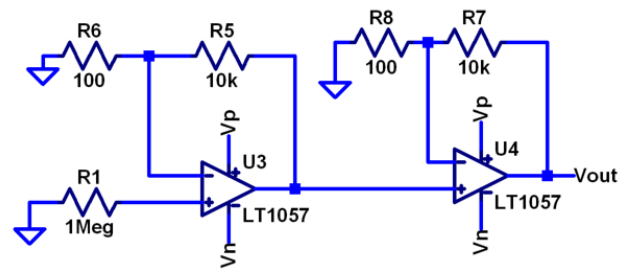

Fig. 13: A 1M resistor serves as a predictable noise source, which is then amplified to a usable level by a low-noise operational amplifier.

effects can be modelled and compensated for later. But noise is the one imperfection that cannot.

\section{A Laboratory Noise Source}

A calibrated noise generator functions as a "world's worst sensor", that emulates the noise of a sensor without actually sensing anything. Such a generator allows a signal chain's response to noise to be measured directly. The circuit shown in Figure 13 uses a $1 \mathrm{M}$ resistor as a $127 \mathrm{nV} / \sqrt{\mathrm{Hz}}$ (at room temperature) noise source with "okay accuracy" and bandwidth. While the accuracy is only "okay", this method has advantages:

- It is based on first principles, so in a sense can act as an uncalibrated standard.

- It is truly random, with no repeating patterns.

The OP482 is an ultralow bias current amplifier with correspondingly low current noise, and a voltage noise low enough that the noise due to a $1 \mathrm{M}$ input impedance is dominant. Configured with a gain of 2121 , the output noise is $269 \mu \mathrm{V} / \sqrt{\mathrm{Hz}}$.

The noise source was verified with an ADALM2000 USB instrument, using the Scopy ${ }^{11}$ GUI's spectrum analyzer, shown in Figure 14.

Under the analyzer settings shown, the ADALM2000 noise floor is $40 \mu \mathrm{V} / \sqrt{\mathrm{Hz}}$, well below the $269 \mu \mathrm{V} / \sqrt{\mathrm{Hz}}$ of the noise source.

While Scopy is useful for single, visual measurements, the functionality can be replicated easily with the SciPy periodogram function. Raw data is collected from an ADALM2000 using the libm $2 \mathrm{k}^{12}$ and Python bindings, minimally processed to remove DC content (that would otherwise "leak" into low frequency bins), and scaled to $\mathrm{nV} / \sqrt{\mathrm{Hz}}$. This method, shown in the Noise Source Measurement code block can be applied to any data acquisition module, so long as the sample rate is fixed and known, and data can be formatted as a vector of voltages. 


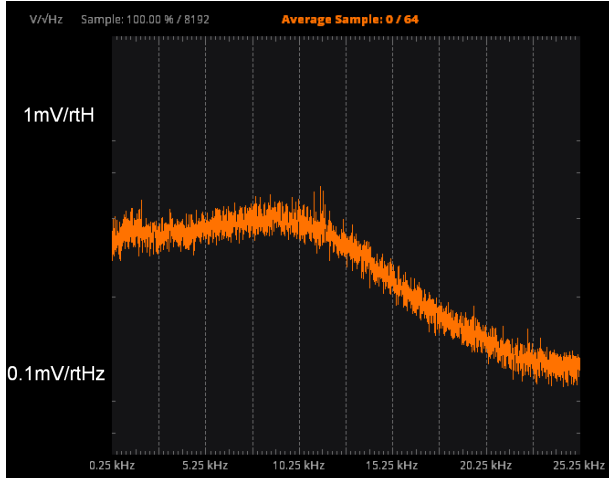

Fig. 14: The output of the resistor-based laboratory noise generator has a usable bandwidth of approximately $10 \mathrm{kHz}$.

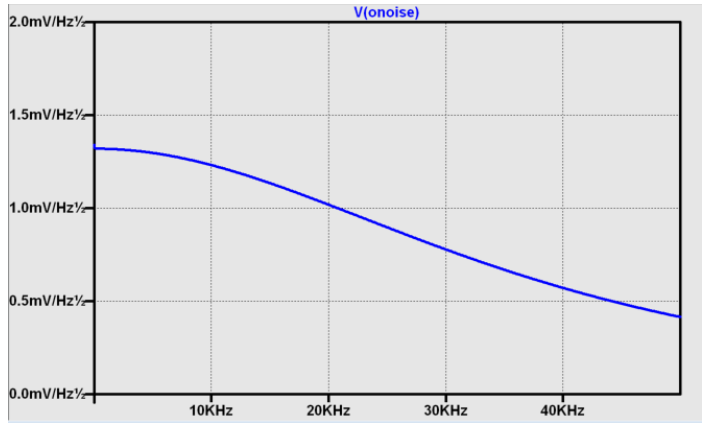

Fig. 15: An LTspice simulation of the laboratory noise source shows approximately the same usable bandwidth as the measured circuit.

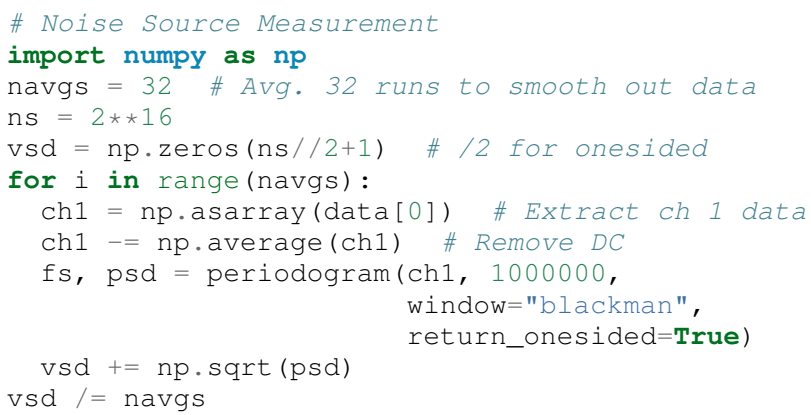

We are now armed with a known noise source and a method to measure said source, both of which can be used to validate signal chains.

\section{Modeling Signal Chains in LTspice}

LTspice is a freely available, general-purpose analog circuit simulator that can be applied to signal chain design. It can perform transient analysis, frequency-domain analysis (AC sweep), and noise analysis, the results of which can be exported and incorporated into mixed signal models using Python.

Figure 15 shows a noise simulation of the analog noise generator, with close agreement to experimental results. An op-amp with similar properties to the OP482 was used for the simulation.

Figure 15 circuit's noise is fairly trivial to model, given that it is constant for some bandwidth (in which a signal of interest would lie), above which it rolls off with approximately a first order lowpass response. Where this technique comes in handy is modeling non-flat noise floors, either due to higher order analog filtering, or active elements themselves. The classic example is the

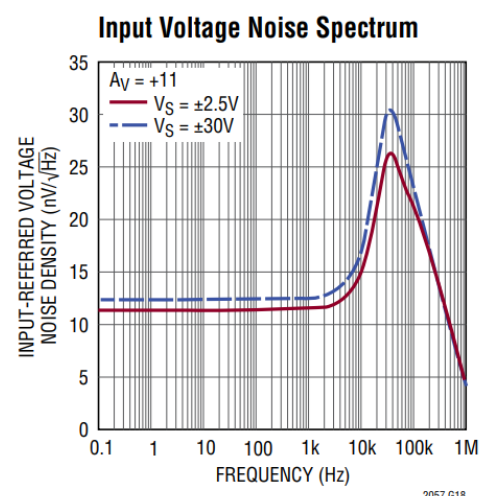

Fig. 16: The LTC2057 noise density is flat at low frequencies, with a peak at $50 \mathrm{kHz}$ (half of the internal oscillator's $100 \mathrm{kHz}$ frequency).

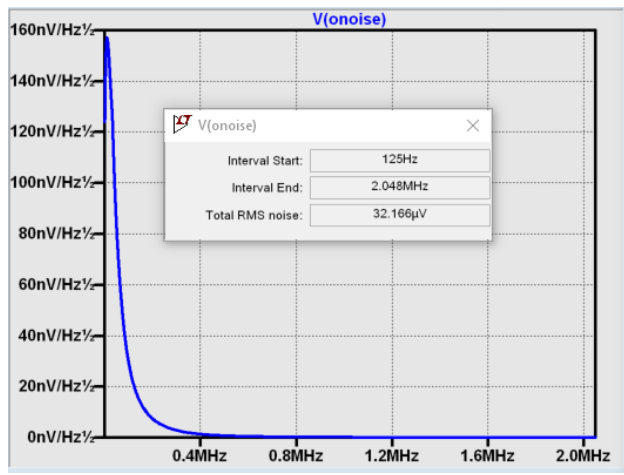

Fig. 17: LTspice is used to simulate the output nosie of an LTC2057 in a noninverting gain of +10 configuration. LTspice provides simple tools for integrating noise, but results of any simulation can be exported and imported into Python for further analysis.

"noise mountain" that often exists in autozero amplifiers such as the LTC2057, as seen in Figure 16 , from Ref. ${ }^{13}$.

Importing LTspice noise data for frequency domain analysis in Python is a matter of setting up the simulation command such that exact frequencies in the analysis vector are simulated. In this case, the noise simulation is set up for a simulation with a maximum frequency of $2.048 \mathrm{MHz}$ and resolution of $62.5 \mathrm{~Hz}$, corresponding to the first Nyquist zone at a sample rate of 4.096 MSPS. Figure 17 shows the simulation of the LT2057 in a non-inverting gain of 10 , simulation output, and exported data format.

In order to determine the impact of a given band of noise on a signal (signal to noise ratio) the noise is root-sum-square integrated across the bandwidth of interest. In LTspice, plotted parameters can be integrated by setting the plot limits, then control-clicking the parameter label. The total noise over the entire $2.048 \mathrm{MHz}$ simulation is $32 \mu \mathrm{VRMS}$. A function to implement this operation in Python is shown in the Integrate Power Spectral Density code block.

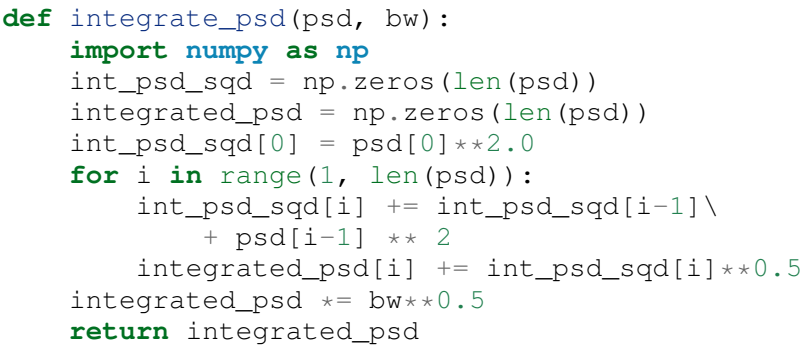


Reading in the exported noise data and passing to the integrate_psd function results in a total noise of $3.21951 \mathrm{e}-05$, very close to LTspice's calculation.

\section{Generating Test Noise}

Expanding on the functionality of the purely analog noise generator above, it is very useful to be able to produce not only flat, but arbitrary noise profiles - flat "bands" of noise, "pink noise", "noise mountains" emulating peaking in some amplifiers. The Generate Time-series From Half-spectrum code block starts with a desired noise spectral density (which can be generated manually, or taken from an LTspice simulation), the sample rate of the time series, and produces a time series of voltage values that can be sent to a DAC.

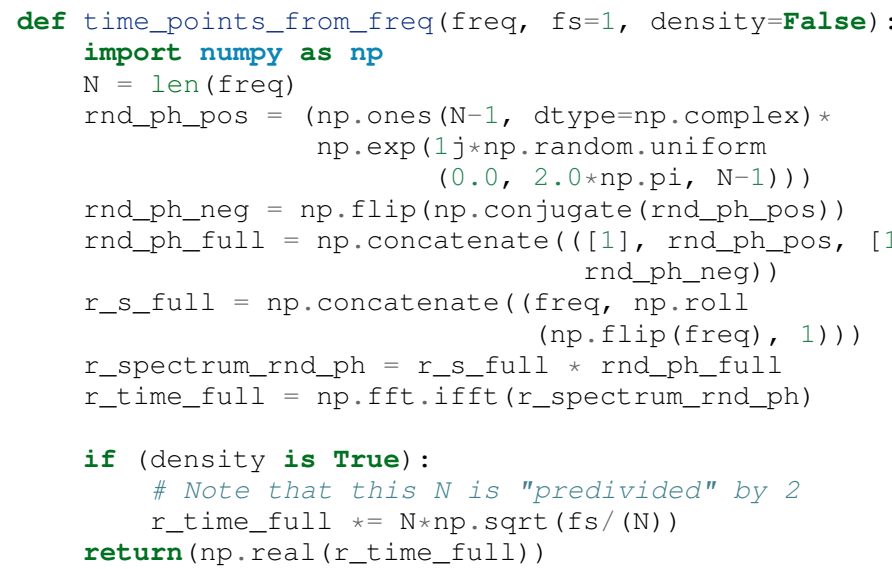

This function can be verified by controlling one ADALM2000 through a libm2k script, and verifying the noise profile with a second ADALM2000 and the spectrum analyzer in the Scopy GUI. The Push Noise Time-series to ADALM2000 code snippet generates four "bands" of $1 \mathrm{mV} / \sqrt{\mathrm{Hz}}$ noise on the ADALM2000 W2 output (with a sinewave on W1, for double-checking functionality.)

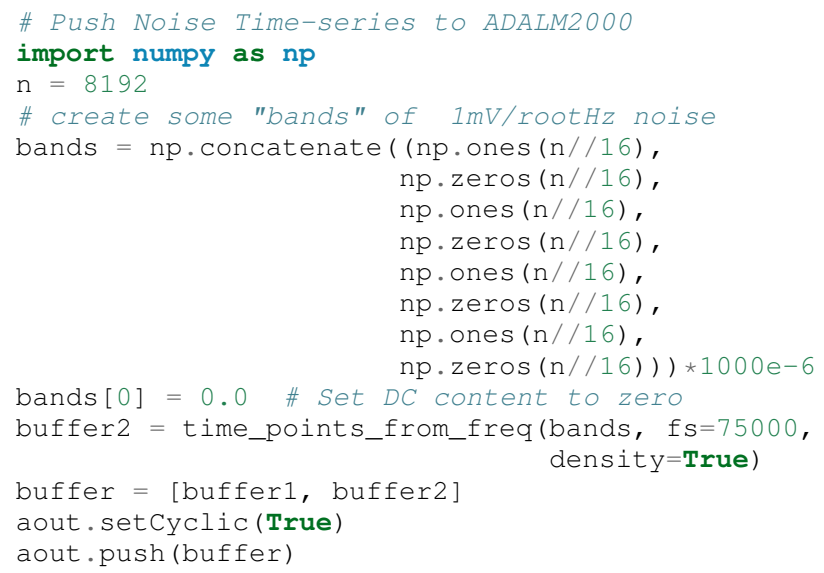

Figure 18 shows four bands of $1 \mathrm{mV} / \sqrt{\mathrm{Hz}}$ noise being generated by one ADALM2000. The input vector is 8192 points long at a sample rate of $75 \mathrm{ksps}$, for a bandwidth of $9.1 \mathrm{~Hz}$ per point. Each "band" is 512 points, or $4687 \mathrm{~Hz}$ wide. The rolloff above $\sim 20 \mathrm{kHz}$ is the SINC rolloff of the DAC. If the DAC is capable of a higher sample rate, the time series data can be upsampled and filtered by an interpolating filter ${ }^{14}$.

This noise generator can be used in conjunction with the pure analog generator for verifying the rejection properties of a signal chain.

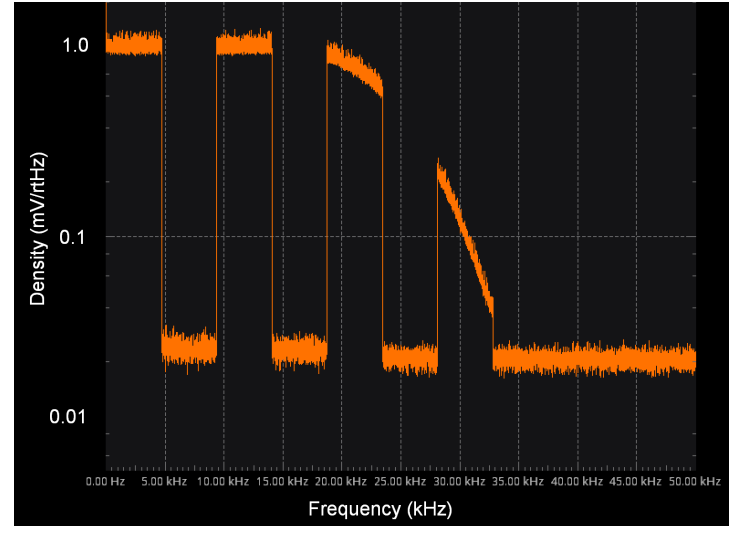

Fig. 18: The Scopy spectrum analyzer is used to verify the arbitrary noise generator. Deep notches between noise bands expose the analyzer's noise floor, showing that an arbitrary noise profile can be accurately generated.

\section{Modeling and verifying ADC Noise Bandwidth}

1] 'External noise sources and spurious tones above Fs/2 will fold back (alias) into the DC-Fs/2 region - and a converter may be sensitive to noise far beyond Fs/2 - the AD872A mentioned above has a sample rate of $10 \mathrm{Msps}$, but an input bandwidth of $35 \mathrm{MHz}$. While performance may not be the best at such high frequencies, this converter will happily digitize 7 Nyquist zones of noise and fold them back on top of your signal. This illustrates the importance of antialias filters for wideband ADCs. But converters for precision applications, which are typically sigma-delta (like the AD7124-8) or oversampling SAR architectures, in which the input bandwidth is limited by design.

It is often useful to think of the "equivalent noise bandwidth" (ENBW) of a filter, including an ADC's built-in filter. The ENBW is the bandwidth of a flat passband "brick wall" filter that lets through the same amount of noise as the non-flat filter. A common example is the ENBW of a first-order R-C filter, which is:

$$
E N B W=f c * \pi / 2
$$

Where $f c$ is the cutoff frequency of the filter. If broadband noise, from "DC to daylight", is applied to the inputs of both a $1 \mathrm{KHz}$, first-order lowpass filter and $1.57 \mathrm{kHz}$ brickwall lowpass filter, the total noise power at the outputs will be the same.

The ENBW Example code block accepts a filter magnitude response, and returns the effective noise bandwidth. A single-pole filter's magnitude response is calculated, and used to verify the $\mathrm{ENBW}=f c * p i / 2$ relationship.

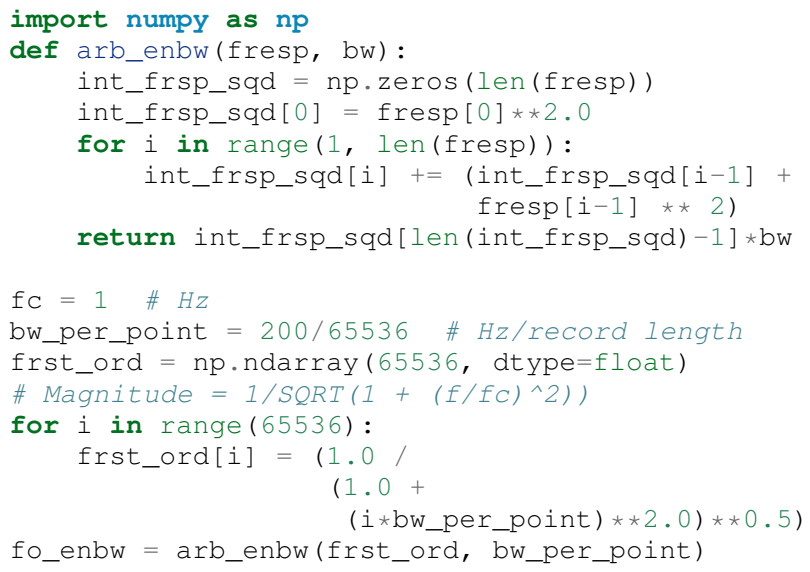



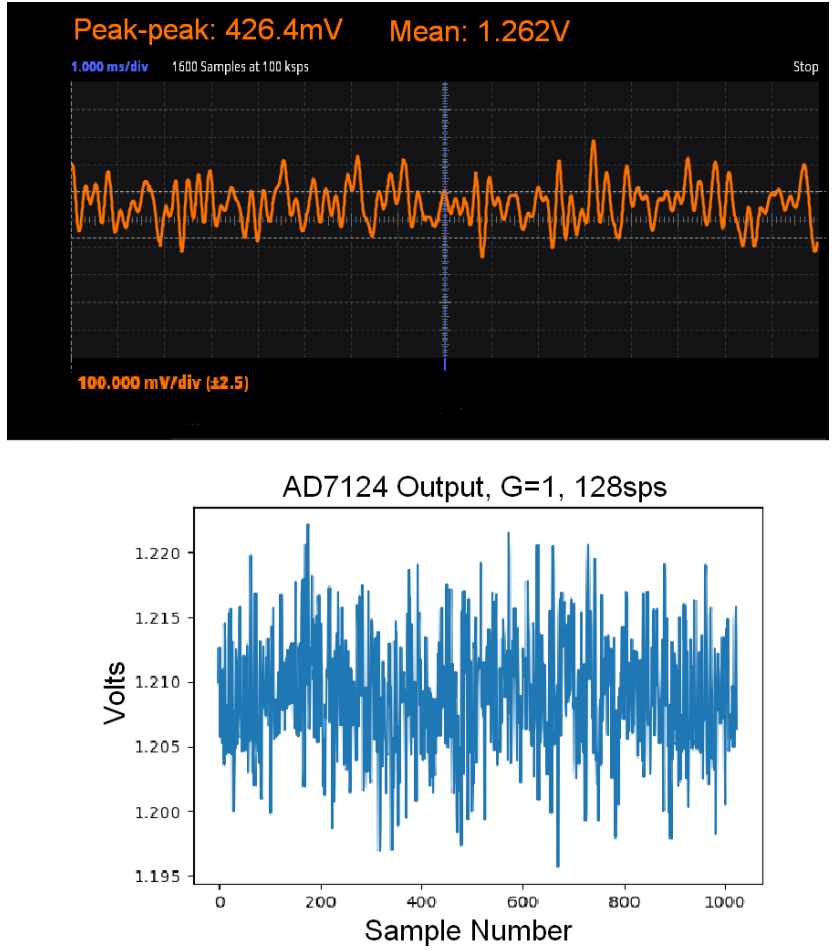

Fig. 19: A $1 \mathrm{mV} / \sqrt{\mathrm{Hz}}$ noise band is driven into the AD7124-8 input. A qualitative reduction in noise is apparent; $426 \mathrm{mV}$ peak-to-peak noise at the ADC input results in approximately $25 \mathrm{mV}$ peak-to-peak noise at the ADC output. The $5.1 \mathrm{mVRMS}$ total output noise is close to the predicted $5.69 \mathrm{mVRMS}$, given the $1 \mathrm{mV} / \sqrt{\mathrm{Hz}}$ noise density and $31 \mathrm{~Hz}$ ENBW of the ADC's filter.

This function can be used to calculate the ENBW of an arbitrary filter response, including the AD7124's internal filters. The frequency response of the AD7124 SINC4 filter, 128sps sample rate can be calculated similar to the previous $50 / 60 \mathrm{~Hz}$ rejection filter example. The arb_anbw function returns a ENBW of about $31 \mathrm{~Hz}$.

The ADALM2000 noise generator can be used to validate this result. Setting the test noise generator to generate a band of $1000 \mu \mathrm{V} / \sqrt{\mathrm{Hz}}$ should result in a total noise of about 5.69mVRMS, and measured results are approximately 5.1mVRMS total noise. The oscilloscope capture of the ADC input signal is plotted next to the ADC output data, in Figure 19. Note the measured peak-topeak noise of $426 \mathrm{mV}$, while the ADC peak-to-peak noise is about $26 \mathrm{mV}$. While such a high noise level is (hopefully) unrealistic in an actual precision signal chain, this exercise demonstrates that the ADC's internal filter can be relied on to act as the primary bandwidth limiting, and hence noise reducing, element in a signal chain.

\section{Conclusion}

Noise is a limiting factor in any signal chain; once noise contaminates a signal, information is lost. Before building a signal acquisition system, the application requirements must be understood, components selected accordingly, and the prototype circuit tested. This tutorial offers a collection of methods that accurately model and measure sensor and signal chain noise that can be used during the design and testing process.

The techniques detailed in this tutorial are, individually, nothing new. However, in order to achieve an adequate system, it becomes valuable to have a collection of fundamental, easy to implement, and low-cost techniques to enable signal chain modeling and verification. Even though industry continues to offer parts with increased performance, there will always be a certain limitation that one must be aware of. These techniques can not only be used to validate parts before building a mixed-mode signal chain, but also to identify design faults in an existing one.

\section{Acknowledgements}

- Jesper Steensgaard, who enabled/forced a paradigm shift in thinking about signal chain design, starting with the LTC2378-20.

- Travis Collins, Architect of Pyadi-iio (among many other things).

- Adrian Suciu, Software Team Manager and contributor to libm2k.
1. "Converter Connectivity Tutorial", https://wiki.analog.com/university/ labs/software/iio_intro_toolbox, accessed 1 July, 2021.

2. Analog Devices Education Tools Repository https://doi.org/10.5281/ zenodo.5105696

3. Pauli Virtanen, Ralf Gommers et al. (2020) SciPy 1.0: Fundamental Algorithms for Scientific Computing in Python. Nature Methods, 17(3), 261272.

4. "LTspice Simulator", https://www.analog.com/en/design-center/designtools-and-calculators/ltspice-simulator.html, accessed 1 July, 2021.

5. Smith, Steven W, The Scientist \& Engineer's Guide to Digital Signal Processing, https://www.analog.com/en/education/education-library/scientist_ engineers_guide.html, accessed 1 July, 2021.

6. Man, Ching, "Quantization Noise: An Expanded Derivation of the Equation, SNR = $6.02 \mathrm{~N}+1.76 "$, https://www.analog.com/media/en/trainingseminars/tutorials/MT-229.pdf, accessed 1 July, 2021.

7. Kester, Walt, "Taking the Mystery out of the Infamous Formula, "SNR $=6.02 \mathrm{~N}+1.76 \mathrm{~dB} "{ }^{\prime \prime}$ Analog Devices Tutorial, 2009, https://www.analog.com/ media/en/training-seminars/tutorials/MT-001.pdf, accessed 1 July, 2021.

8. "AD7124-8 Rev E" https://www.analog.com/media/en/technicaldocumentation/data-sheets/ad7124-8.pdf, accessed 1 July, 2021.

9. Charles R. Harris, K. Jarrod Millman, et al. Array programming with NumPy, Nature, 585, 357-362 (2020) DOI:10.1038/s41586-020-2649-2

10. "pyadi-iio: Device Specific Python Interfaces For IIO Drivers", https: //wiki.analog.com/resources/tools-software/linux-software/pyadi-iio, accessed 1 July, 2021.

11. "Scopy", https://wiki.analog.com/university/tools/m2k/scopy, accessed 1 July, 2021.

12. "What is Libm2k?", https://wiki.analog.com/university/tools/m2k/ libm2k/libm2k, accessed 1 July, 2021.

13. "LTC2057/LTC2057HV High Voltage, Low Noise Zero-Drift Operational Amplifier", https://www.analog.com/media/en/technical-documentation/ data-sheets/2057f.pdf, accessed 1 July, 2021.

14. Kester, Walt, "Oversampling Interpolating DACs", Analog Devices Tutorial, 2009, https://www.analog.com/media/en/training-seminars/tutorials/ MT-017.pdf, accessed 1 July, 2021.

15. Ruscak, Steve and Singer, L, "Using Histogram Techniques to Measure ADC Noise" Analog Dialogue, Volume 29, May, 1995. https://www.analog.com/en/analog-dialogue/articles/histogram-techniquesmeasure-adc-noise.html, accessed 1 July, 2021. 